\title{
Crystalline-Size Dependence of Dual Emission Peak on Hybrid Organic Lead-lodide Perovskite Films at Low Temperatures
}

\author{
Raquel Chulia-Jordan, ${ }^{* \dagger ®}$ Elena Mas-Marzá, ${ }^{\ddagger}$ Alfredo Segura, ${ }^{\S}$ Juan Bisquert, ${ }^{\ddagger} \|_{\odot}$ \\ and Juan P. Martínez-Pastor ${ }^{\dagger}$ \\ †Instituto de Ciencia de los Materiales, Universitat de València, c/Catedrático J. Beltrán, 2, Paterna 46980, Spain \\ ${ }^{\ddagger}$ Institute of Advanced Materials, Universitat Jaume I, 12006 Castelló, Spain \\ ${ }^{\S}$ Departamento de Física Aplicada-ICMUV, Universitat de València, C/Dr. Moliner 50, 46100 Burjassot, Spain \\ "Division of Physical Sciences and Engineering, King Abdullah University of Science and Technology (KAUST), Thuwal \\ 23955-6900, Kindom of Saudi Arabia
}

\begin{abstract}
In this work, we have investigated the crystalline-size dependence of optical absorption and photoluminescence emission of $\mathrm{CH}_{3} \mathrm{NH}_{3} \mathrm{PbI}_{3}$ films, which is necessary to identify the potential practical applications of the gadgets based on perovskite films. This study was carried out at low temperatures to minimize the extra complexity induced by thermal effects. The purpose was to clarify the origin of the dual emission peak previously reported in the literature. We found that the grain size is responsible for the appearance or disappearance of this dual emission on $\mathrm{CH}_{3} \mathrm{NH}_{3} \mathrm{PbI}_{3}$ at low temperatures, whereas we have inferred that the thickness of the perovskite layer is a much more important factor than the size of the grains in the location of the energy of the band gap. Moreover, the increase in the grain size allows slowing down the phase transition. Additionally, we evidence a decrease in the effective Rydberg energy of the exciton in several samples, from $23-25 \mathrm{meV}$ at $7 \mathrm{~K}$ to

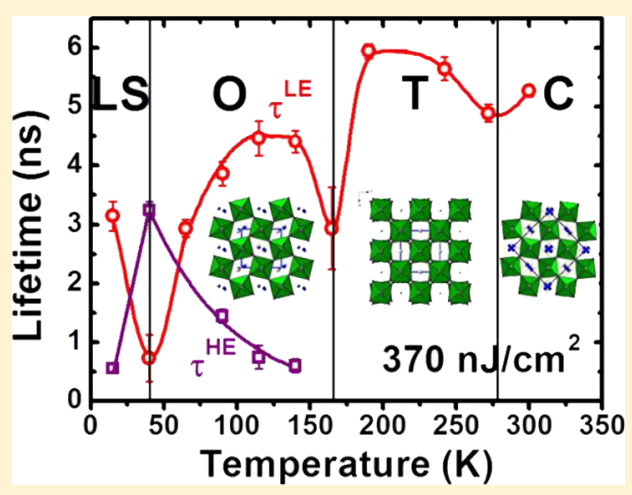
$12-13 \mathrm{meV}$ at $165 \mathrm{~K}$, by fitting to Elliott-Toyozawa theory. We have extracted other important physical parameters of perovskites from the photoluminescence-data deconvolution, such as the band gap, exciton-phonon interaction, and exciton binding energy. A new phase transition at $45.5 \mathrm{~K}$ was determined by the temperature dependence of full width at half-maximum and the integrated intensity of the photoluminescence, and it was confirmed by the radiative lifetime obtained from the time-resolved photoluminescence emission by means of time-correlated single-photon counting at different temperatures, excitation fluencies, and emission energies.
\end{abstract}

\section{INTRODUCTION}

Recently, a significant effort to understand the photophysical processes occurring in perovskites has been carried out because of the potential that these materials offer to practical applications, such as solar cells, field effect transistors, lasers, batteries, or light-emitting devices. ${ }^{1-5}$ In particular, the organic-inorganic metal-halide perovskite has been widely investigated because of the extraordinary improvement shown on the efficient solar cells. The unique properties of $\mathrm{CH}_{3} \mathrm{NH}_{3} \mathrm{PbI}_{3}$, such as band gap modulation or long-range balanced electron- and hole-diffusion lengths, make it a promising photovoltaic material. ${ }^{6-8}$ The structural stability and polymorphism of this material as a function of temperature $(T)$ has been previously reported: cubic-tetragonal (327 K) and tetragonal-orthorhombic $(165 \mathrm{~K})$. However, there are still some unsolved questions about important photophysical processes occurring in $\mathrm{CH}_{3} \mathrm{NH}_{3} \mathrm{PbI}_{3}$ films that need to be clarified, such as the origin of the dual emission peak already observed at low temperatures. ${ }^{9}$ Although its existence was previously shown, the scientific community has not come to an agreement about the origin of this dual emission. For example,
Xing $^{10}$ reported three emission peaks, which have been assigned to two bound-exciton emissions and a free exciton; however, Fang ${ }^{11}$ attributed the low- and high-energy emission peaks to free and bound excitons, and Kong ${ }^{12}$ attributed them to a donor-acceptor pair (DAP) (low energy) and free-exciton transitions. Last, Panzer ${ }^{13}$ and Wehrenfennig ${ }^{14}$ ascribed the existence of this dual emission to the presence of tetragonal inclusions in the orthorhombic phase at low temperatures, whereas $\mathrm{Dar}^{6}$ attributed it to the coexistence of $\mathrm{CH}_{3} \mathrm{NH}_{3}{ }^{+}$ ordered and $\mathrm{CH}_{3} \mathrm{NH}_{3}{ }^{+}$-disordered orthorhombic domains. Hence, despite the current effort, an understanding of the essential characteristics of perovskite films is still evolving.

Moreover, it has been studied how the thickness of the perovskite films affects the absorbance and the photocurrent. The absorption coefficient curve of the organometal-halide perovskites $\left(\mathrm{CH}_{3} \mathrm{NH}_{3} \mathrm{PbX}_{3}, \mathrm{X}=\right.$ halogen $)$ at room temperature showed not only an unusual sharp shoulder near its reported

Received: July 18, 2018

Revised: September 8, 2018

Published: September 10, 2018 
band gap value $(\sim 1.57 \mathrm{eV})$ but also that this shoulder occurs at higher absorption coefficients than most other comparable semiconductors. This fact clarifies why very thin absorber films are sufficient for perovskite solar cell manufacture. ${ }^{15,16}$ Moreover, because of the purely exponential trend of the Urbach tail, any traces of absorption by deep levels are observed below the absorption bandedge. This influences such high open-circuit voltage $\left(V_{\mathrm{OC}}\right)$ values $^{17}$ (up to $1.13 \mathrm{~V}$ ) comparable to the perovskite band gap.

In the present study, we focus on the nature of the exciton recombination channels below/above the orthorhombictetragonal phase transition $(165 \mathrm{~K})$, depending on the crystalline size of perovskite films, to clarify the origin of the dual emission peak already observed at low temperatures. Previously, it has been verified in other kind of structures that periodicities of the order of $250 \mathrm{~nm}$ can produce duplication of bands. ${ }^{18}$ To that end, we have studied the dependence of the emission and absorption properties of perovskite films on the grain size using time-integrated, time-resolved photoluminescence (TRPL) spectroscopy and by measuring the absorbance curves and photocurrent on the cell. We have found that the grain size is responsible for the appearance or disappearance of dual emission on $\mathrm{CH}_{3} \mathrm{NH}_{3} \mathrm{PbI}_{3}$ at low temperatures. A minimum size is required to observe both the unusual blue shift of the band gap and the dual emission reported in perovskite materials. ${ }^{6}$ Moreover, the dependence of photoluminescence (PL) spectra on temperature and excitation wavelengths was also determined. Our findings provide new insights into the photophysical processes occurring in perovskite films.

\section{METHODS AND MATERIALS}

2.1. Sample Preparation and Description. Sample preparations have been previously explained. ${ }^{19}$ Fluorinedoped tin oxide (FTO) coated glass was carefully cleaned with ultrapure water and ethanol. Then, the substrates were dried using a $\mathrm{N}_{2}$ stream. $A \approx 40 \mathrm{~nm}$-thick layer of $\mathrm{TiO}_{2}$ was deposited on the FTO-coated glass by spin-coating anhydrous ethanol solution of titanium isopropoxide and heated at 500 ${ }^{\circ} \mathrm{C}$ for $30 \mathrm{~min}$ under room atmospheric conditions. On this $\mathrm{TiO}_{2}$ buffer layer was deposited a $200 \mathrm{~nm} \mathrm{TiO}$ mesoporous layer. Halide perovskite $\mathrm{CH}_{3} \mathrm{NH}_{3} \mathrm{PbI}_{3}$ "cuboidlike" films of 350 $\mathrm{nm}(\mathrm{S} 1)$ and $300 \mathrm{~nm}$ (S2) thickness were deposited inside a glovebox by spin-coating $30 \mu \mathrm{L}$ of a $1.082 \mathrm{M}$ solution of $\mathrm{PbI}_{2}$ in dimethylformamide at $500 \mathrm{rpm}$ for $5 \mathrm{~s}$ and $6000 \mathrm{rpm}$ for 20 $\mathrm{s}$, followed by heating at $40{ }^{\circ} \mathrm{C}$ for $3 \mathrm{~min}$ and $100{ }^{\circ} \mathrm{C}$ for 10 min on a hot plate. The $\mathrm{PbI}_{2}$ film was then dipped for $1 \mathrm{~min}$ in a $\mathrm{CH}_{3} \mathrm{NH}_{3} \mathrm{I}$ solution in isopropanol $(0.044 \mathrm{M}$ or $0.063 \mathrm{M}$ depending on the grain size), spun at $500 \mathrm{rpm}$ for $5 \mathrm{~s}, 1500$ $\mathrm{rpm}$ for $10 \mathrm{~s}$, and $3000 \mathrm{rpm}$ for $20 \mathrm{~s}$, and heated at $40{ }^{\circ} \mathrm{C}$ for 3 min and at $100{ }^{\circ} \mathrm{C}$ for $10 \mathrm{~min}$ on a hot plate.Scanning electron microscopy (SEM) images of the substrate were obtained using a JSM-7000F JEOL 216 FEG-SEM system using secondary and retrodispersed electron beams (Figure 1).

2.2. Experimental Setup. Samples have been characterized by placing them in the cold finger of a commercial closed cycle, compressed helium cryostat (ARS DE-202). This cryogenerator has a heating resistance and a thermometer, with which the temperature can be controlled from $10 \mathrm{~K}$ to room temperature. For time-integrated PL measurements we used a continuos wave laser diode at $405 \mathrm{~nm}$. In time-resolved PL (TRPL) experiments we used for excitation a 200 fs pulsed Ti:sapphire (Coherent Mira 900D, $76 \mathrm{MHz}$ of repetition rate)
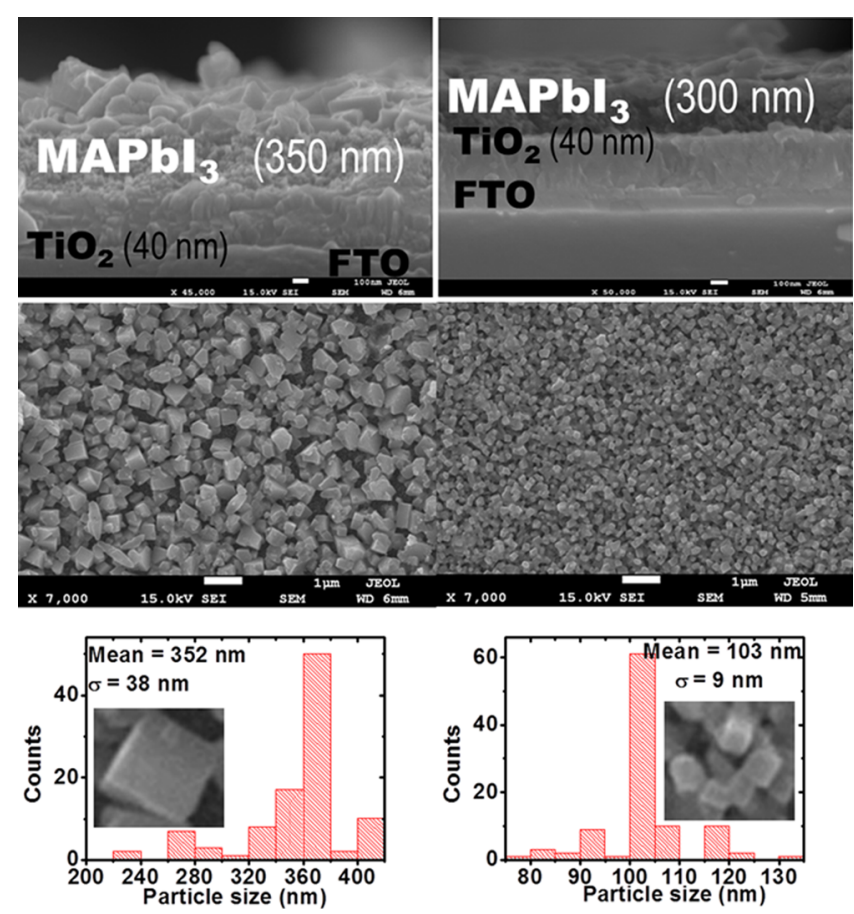

Figure 1. (From up to down) Thickness of the perovskite samples, homogeneity in terms of their dimensions and particle sizes (mean of the dimensions of the particles and their standard deviation, $\sigma$ ). The structural and morphological characterizations were obtained by using a JSM-7000F JEOL 216 FEG-SEM system. (Left) Particle size 352 nm. (Right) Particle size $103 \mathrm{~nm}$.

laser doubled to $400 \mathrm{~nm}$ with a BBO crystal. The backscattered PL signal was dispersed by a double $0.3-\mathrm{m}$ focal length grating spectrograph/spectrometer $(1200 \mathrm{~g} / \mathrm{mm}$ with $750 \mathrm{~nm}$ blaze $)$ and detected by an Andor Newton 970 EMCCD camera (for time-integrated PL spectra) and by a Si Micro Photon Device (MPD) single photon avalanche diode (SPAD) photodetector connected through a multimode optical fiber to the monochromator (for time-resolved PL spectra); the SPAD was attached to a time correlated single photon counting electronic board (TCC900 from Edinburgh Instruments). 20,21 Absorbance spectra were registered by using a halogen lamp focused on the sample and an Ocean Optics minispectrometer.

\section{RESULTS AND DISCUSSION}

To reach a deeper understanding of the underlying physical mechanisms of $\mathrm{CH}_{3} \mathrm{NH}_{3} \mathrm{PbI}_{3}$ (hereafter $\mathrm{MAPbI}_{3}$ ) films, we have investigated the crystalline-size dependence of optical absorption and PL emission. It has been previously seen that this is an important factor in absorption phenomena. ${ }^{22,23}$ The accurate control of the crystalline size and shape of particles that constitute the perovskite films helped us to eliminate the random signals due to the surface morphology of spatially inhomogeneous samples. ${ }^{12,24,25}$ Moreover, the dependence of PL spectra on temperature and excitation wavelengths was also determined.

3.1. Optical Absorption: Exciton Binding Energy and $E_{\mathrm{g}}$ in $\mathrm{MAPbl}_{3}$. The evolution of the absorbance curves in small- and large-grain samples with temperature, that is, for grains of two different average sizes (352 and $103 \mathrm{~nm}$, hereafter also denoted as $\mathrm{S} 1$ and $\mathrm{S} 2$, respectively), of $\mathrm{MAPbI}_{3}$ was measured. 
As shown in Figure 2, the material with smaller grains transforms from the tetragonal into orthorhombic phase at $T_{c}$
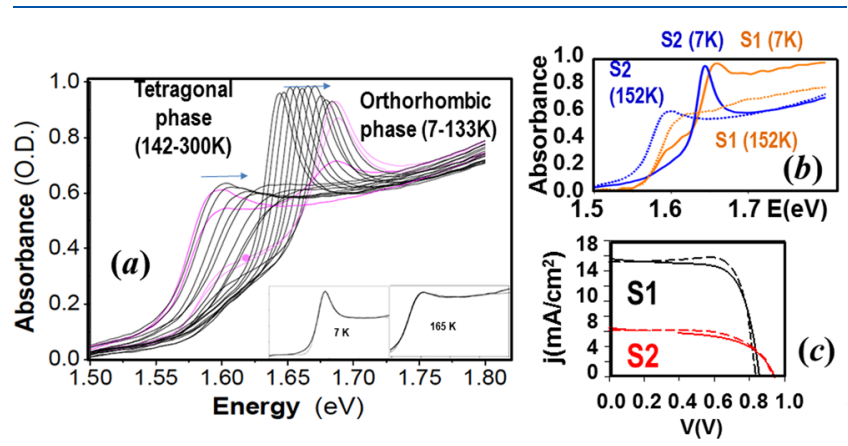

Figure 2. (a) Evolution of the absorbance with temperature in smaller $\mathrm{MAPbI}_{3}$ grains. Transition phase around $150 \mathrm{~K}$. Inset: Fitting using Elliott-Toyozawa theory with Lorentzian (low $T$ region) and Gaussian (high $T$ region) broadenings. (Left inset) Orthorhombic phase at $T=7 \mathrm{~K}$ with an effective Rydberg energy of $23-25 \mathrm{meV}$. (Right inset) Tetragonal phase at $T=165 \mathrm{~K}$ with an effective Rydberg energy of $12-13 \mathrm{meV}$. (b) Normalized absorbance curves of larger and smaller grain samples (denoted as S1 and S2, respectively) at two different temperatures, 7 and $152 \mathrm{~K}$ (phase transition). (c) JV curves. Cell devices prepared under the same conditions as perovskites $S 1$ and S2. Dashed line: from 1.2 to $-0.1 \mathrm{~V}$. Continuous line: from -0.1 to $1.2 \mathrm{~V}$.

$=150 \mathrm{~K}$, and both phases undoubtedly coexist below $T_{\mathrm{c}}$ down to approximately $110 \mathrm{~K}$ (see the inset of Figure $2 \mathrm{a}$ ). ${ }^{26}$ The absorbance spectra clearly show an excitonic absorbance resonance in both phases:

(i) For the orthorhombic low- $T$ phase, the exciton resonance is clearer and fairly narrower as temperature decreases (down to $7 \mathrm{~K}$ ). It has Lorentzian rather than Gaussian broadening.

(ii) In the case of the tetragonal high- $T$ phase, the excitonic resonance remains up to about $220 \mathrm{~K}$; above this temperature, it is being only observed as a shoulder (shallow maximum).

It must be noted that the low-energy part of the spectrum is purely excitonic, whereas the high-energy part presents the continuous excitonic absorbance or band-band.

For the sake of comparison, Figure $2 \mathrm{~b}$ shows the absorbance curves of the samples with different grain sizes at two temperatures, 7 and $152 \mathrm{~K}$ (temperature at which the orthorhombic to tetragonal transition occurs). It can be clearly seen that the excitonic front is somewhat wider and shifted toward a high energy in sample S1 (larger grains) in relation to sample S2 (smaller grains), which is consistent with the corresponding larger photocurrent measured on the cell, due to light trapping effect by scattering (Figure 2c). The smaller open circuit voltage measured in S1 might be due to a greater disorder in this film.

By fitting the absorbance spectra using the ElliottToyozawa theory with Lorentzian (low $T$ region) and Gaussian (high $T$ region) broadenings (see Figure 2a), the evolution for the orthorhombic phase represented by blue circles and the evolution for the tetragonal phase represented by red squares (the points between 240 and $300 \mathrm{~K}$ are less precise because the maximum cannot be unequivocally distinguished) can be obtained. The shift of the excitonic peak with $T$ has a positive rate (contrary to that observed in $\mathrm{Si}$, $\mathrm{Ge}$, and III-V semiconductors and II-VI) on the order of
$0.4-0.5 \mathrm{meV} / \mathrm{K}$ in both phases (see Figure $3 \mathrm{a}$ for exact fitted values). These values are very similar to those measured in $\mathrm{PbS}$
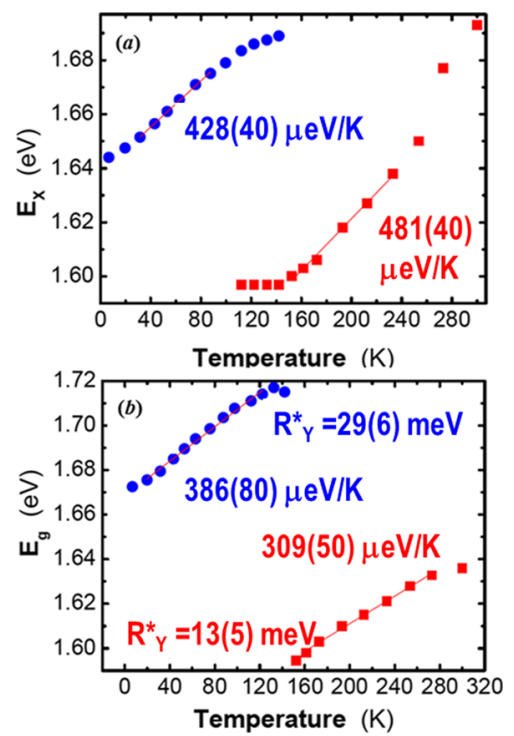

Figure 3. Temperature evolution of the excitonic optical transition (a) and band gap energy (b). Their temperature rates are indicated in both panels.

and $\mathrm{PbSe}$ semiconductors and are due to $\mathrm{Pb}$ orbitals contributing to the valence band of these materials. Fits of spectra in Figure 2a also confirms the decrease in the effective Rydberg energy of the exciton from 29(6) to 13(5) meV after the phase transition temperature, which is consistent with recent estimations. ${ }^{27}$ In old reports, $35-50 \mathrm{meV}$ was estimated from magneto-PL measurements. ${ }^{28,29}$

After Toyozawa, Lorentzian/Gaussian broadening is related to weak/strong phonon coupling. By fitting the full excitonic curves, we could also determine the dependence of the energy of the band gap, $E_{g}$, on $T$ for both phases (see Figure $3 b$ ). The observed variation is fairly linear, and the values of the $E_{\mathrm{g}}$ slopes are shown in the figure. The slopes are lower than those obtained for the excitonic binding energy but similar to those observed in some IV-VI semiconductors.

3.2. Time-Integrated PL: Band Studies. Figure 4 shows the time-integrated $\mathrm{PL}$ spectra of a perovskite $\left(\mathrm{MAPbI}_{3}\right)$ film consisting of larger grains (average size $352 \mathrm{~nm}$ ) at temperatures ranging from $15 \mathrm{~K}$ up to $293 \mathrm{~K}$ and using

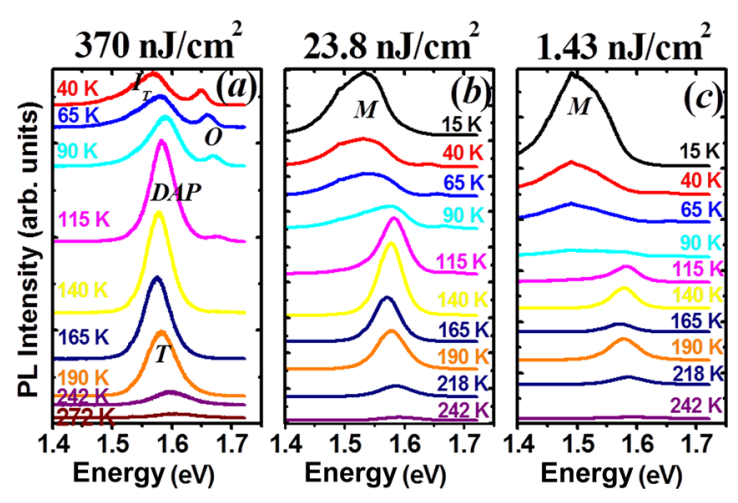

Figure 4. Time-integrated temperature-dependent PL spectra of large-grain films of perovskite at three different excitation fluences: 370 (a), 23.8 (b), and $1.43 \mathrm{~nJ} / \mathrm{cm}^{2}$ (c). 
three different excitation fluences. As previously reported in the literature, we observed two bands in the spectra, and their behavior can be described as follows:

(i) Traditionally, the higher energy band has been associated with the orthorhombic phase $(\mathrm{O})$. This higher energy emission peak " $O$ " can only be observed at high-fluence excitation (see Figure 4a), and it gradually appears below $140 \mathrm{~K}$, although it is almost imperceptible throughout the whole low-temperature range. This band is placed nearby the corresponding absorption onset. ${ }^{14}$ According to the polaronic mechanism, the spectral width of this band does not decrease with temperature, leading to a characteristic and enduring finite line width, even at very low temperatures. ${ }^{30,31}$

(ii) The low-energy band, on the other hand, has been associated with the tetragonal phase and high-fluence excitation; it is perceptible throughout the whole measured temperature range, even at a low temperature range, causing the appearance of dual emission (see Figure 4). There is a remarkable predominance of the low-energy PL band for $T=140 \mathrm{~K}\left(23.8 \mathrm{~nJ} / \mathrm{cm}^{2}\right)$ as well as for the transition region at $T=115 \mathrm{~K}\left(370 \mathrm{~nJ} / \mathrm{cm}^{2}\right)$. However, the shape of the PL emission in this tetragonal phase (" $T$ ") is independent of the fluence excitation, excluding contributions of defect broadening. Some authors have assigned the lower energy band of this dual emission at high-fluence excitation: (i) to a DAP around the transition ${ }^{12}$ and (ii) to the inclusion of the tetragonal phase $\left(\mathrm{I}_{\mathrm{T}}\right)$ at temperatures below the phase transition. ${ }^{13,14,26,32}$ Therefore, residual tetragonal $\mathrm{MAPbI}_{3}$ crystals could coexist and be responsible for this dual emission. One of the arguments in favour of the inclusion of the tetragonal phase at high-fluence excitation is that at temperatures below the phase transition, we still detect emission centered on the same energy as in the room-temperature phase. ${ }^{14}$ Recently, it has been pointed out about the importance of the nature of the organic cation $\left(\mathrm{CH}_{3} \mathrm{NH}_{3}^{+}\right)$on the appearance of dual emission, ${ }^{6,33}$ whereas the nature of the halide ion (I, $\mathrm{Br})$ is supposed to have a negligible impact. ${ }^{6}$ In other words, the atomic ordering in the organic cation takes place progressively when cooling down.

(iii) Last, the S1 sample at low-fluence excitation (see Figure $4 b, c)$ shows important differences with respect to the high-fluence excitation previously reported. We have measured a new sublow-energy band labelled "M." For the lowest excitation power used $\left(1.43 \mathrm{~nJ} / \mathrm{cm}^{2}\right)$, there is a strong red shift at $T<115 \mathrm{~K}$ of the " $\mathrm{M}$ " band (see Figure 5); whereas the energy at which the "T" and "O" emissions are centered almost does not change with decreasing fluence excitation. Moreover, there also exists a strong red shift with decreasing power, the detected emission of " $\mathrm{M}$ " being no longer centered on the similar energy as the room-temperature " $\mathrm{T}$ " phase. Therefore, the band gap energy strongly depends on the fluence excitation on this region.

Moreover, below $45 \mathrm{~K}$, this sublow-energy band labelled "M" seems to change its tendency and present a blue shift with decreasing temperature. Thus, the behavior of this sublowenergy band, which does not appear in the small-grain perovskite film and reaches the maximum in PL intensity at the temperature of $15 \mathrm{~K}$ for the power of $1.43 \mathrm{~nJ} / \mathrm{cm}^{2}$, might

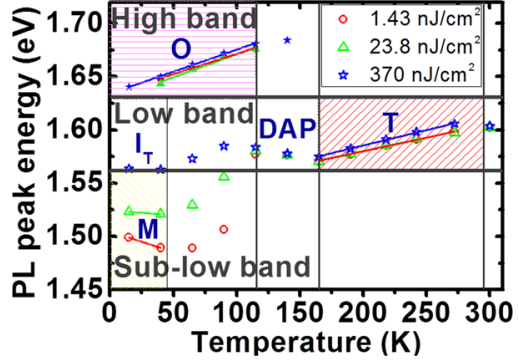

Figure 5. Temperature-dependent data of the PL peak energy of the three bands obtained from the PL spectra. The sample is the larger grain $(352 \mathrm{~nm})$ perovskite film (thickness $350 \mathrm{~nm}$ ). The uncertainty of the data is less than the size of the symbol.

be explained as bound-state excitons ${ }^{10}$ rather than an inclusion of the tetragonal phase.

Hence, a more detailed study of the grain structure should be carried out to gain deeper insight into the dependence of the fundamental properties and processes taking place in this perovskite on the crystalline size.

Figure 5 shows the energy values of the bands as a function of temperature obtained from the PL spectra. By decreasing the temperature, the PL peak energy of the tetragonal band decreases linearly until $T=165 \mathrm{~K}$. Table 1 summarizes the values of the temperature coefficient $\alpha=\partial E / \partial T$ obtained for different fluence excitations.

Table 1. Temperature Coefficient of $\mathrm{CH}_{3} \mathrm{NH}_{3} \mathrm{PbI}_{3}$ Obtained for Different Fluence-Excitations

\begin{tabular}{clcc} 
& \multicolumn{3}{c}{$\alpha=\partial \mathrm{E} / \partial \mathrm{T}(\mu \mathrm{eV} / \mathrm{K})$} \\
\cline { 2 - 4 } power $\left(\mathrm{nJ} / \mathrm{cm}^{2}\right)$ & sub-low & high $=$ orthorhombic & low = tetragonal \\
370 & $-38(7)$ & $370(50)$ & $-38(5)$ \\
23.8 & $-86(12)$ & $24(4)$ & $-86(9)$ \\
1.43 & $-388(70)$ & $1.4(0.2)$ & $-388(60)$ \\
\hline
\end{tabular}

Moreover, the comparison between the calculated ${ }^{34}$ and measured energy band gaps of $\mathrm{MAPbI}_{3}$ is collected on Table 2 . In the temperature range $165 \mathrm{~K}>T>115 \mathrm{~K}$, there exists a transition behaviour before arriving to the orthorhombic structure. Below the tetragonal-to-orthorhombic phase transition, there is a strong dependence of the PL spectra on the fluence excitation, as shown in Figure 4. As the excitation power is decreased, the intensity of the lower energy band remarkably drops, broadens, and shifts to lower energy, whereas the higher energy band nearly vanishes (see Figure 4). Because two coexisting crystallographic phases ${ }^{35-39}$ in several inorganic perovskite materials of mixed ${ }^{38-40}$ and pure ${ }^{41-43}$ composition, including $\mathrm{MAPbI}_{3}$, have been already observed, we suggest that the measured emission in the lowtemperature phase could be explained by supposing a very small relative volume fraction of low-energy sites attributable to small embodiments of individual tetragonal phase crystallites, where photoexcited carriers can be trapped. ${ }^{14}$ Therefore, the subgap sites in the tetragonal crystallites can only be partially filled under weak excitation by the few free carriers. On the other hand, the emission observed at higher energy under strong excitation could be caused by the remaining carriers in the orthorhombic phase after saturating the tetragonal crystallites. Moreover, other arguments in favour of the inclusion of the tetragonal phase at high-fluence excitation are as follows: (i) At temperatures below the phase 
Table 2. Band gap Energy (identified as the $\mathrm{PL}$ peak energy from Gaussian deconvolution) of $\mathrm{CH}_{3} \mathrm{NH}_{3} \mathrm{PbI}_{3}$

\begin{tabular}{lllclc} 
& & \multicolumn{3}{c}{ measured $E_{\mathrm{g} 0}(\mathrm{eV})$} \\
\cline { 3 - 6 } crystal structure & calculated $E_{\mathrm{g} 0}(\mathrm{eV})^{34}$ & $370 \mathrm{~nJ} / \mathrm{cm}^{2}$ & $23.8 \mathrm{~nJ} / \mathrm{cm}^{2}$ & $1.43 \mathrm{~nJ} / \mathrm{cm}^{2}$ & $\mathrm{deconvolution}$ \\
low-symmetry & & $1.564(7)$ & $1.524(3)$ & $1.505(5)$ & $1.491(13)$ \\
orthorhombic & 1.61 & $1.634(7)$ & $1.628(4)$ & $1.633(3)$ & $1.613(4)$ \\
tetragonal & 1.43 & $1.5274(12)$ & $1.529(2)$ & $1.5286(14)$ & $1.535(3)$ \\
cubic & 1.3 & & & $1.415(8)$ \\
\hline
\end{tabular}

transition, we still detect emission centered on the energy similar to that in the room-temperature phase, ${ }^{14}$ in contrast to the increase in the band gap energy measured by optical absorption (see Figure 2). (ii) The shape of the tetragonal band is almost unaffected at temperatures above the phase transition; however the spectral width decreases from $300 \mathrm{~K}$ till $140 \mathrm{~K}$. This is in line with a smaller available phonon population. (iii) The phonon coupling effects could also explain the relatively large width of the tetragonal band. ${ }^{44}$ Nevertheless, we have to keep in mind that these hypotheses are not theoretically verified ${ }^{6}$ and could be unsatisfactory to describe the whole complex phenomenology attached to these perovskite materials. For example, Dar et al. argued that although $\mathrm{CH}_{3} \mathrm{NH}_{3} \mathrm{PbBr}_{3}$ presents dual emission at room temperature, it is not possible to see the existence of tetragonal inclusions in the cubic phase. ${ }^{6}$ Hence, not only an explanation that takes into account all observed trends of the PL spectra over the whole temperature range $(15-300 \mathrm{~K})^{6}$ but also new experiments that allow to identify new dependences in perovskite materials are required.

To confirm the possible physical mechanism for the lowtemperature emission of the $\mathrm{CH}_{3} \mathrm{NH}_{3} \mathrm{PbI}_{3}$ large-grain film, we plot in Figure $6 \mathrm{a}-\mathrm{c}$ the emission normalized to the higher

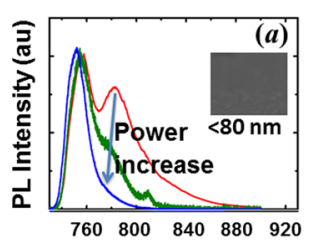

Wavelength $(\mathrm{nm})$

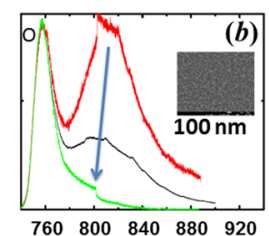

Wavelength $(\mathrm{nm})$

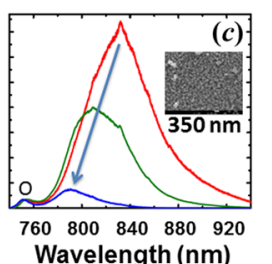

Wavelength $(\mathrm{nm})$
Figure 6. PL intensity depending on the wavelength for three different powers and for a temperature of $40-50 \mathrm{~K}$, well below the phase transition temperature. It corresponds to the sample of a 600 (a), 350 (b), and $300 \mathrm{~nm}$ (c) thick continuous $\mathrm{CH}_{3} \mathrm{NH}_{3} \mathrm{PbI}_{3}$ "cuboidlike" film. The grain sizes are $<80$ (a), 103 (b), and $352 \mathrm{~nm}$ (c). The PL intensity was normalized at the higher energy band. Inset: SEM images of the three spatially homogeneous and conformal films of $\mathrm{CH}_{3} \mathrm{NH}_{3} \mathrm{PbI}_{3}$ perovskite with corresponding crystal sizes.

energy band (orthorhombic phase) at $40 \mathrm{~K}$ (for the $352 \mathrm{~nm}$ thick-grain film), $50 \mathrm{~K}$ (for the $103 \mathrm{~nm}$ thick-grain film), and $50 \mathrm{~K}$ (for the $<80 \mathrm{~nm}$ thick-grain film), respectively. From the comparison to the excitation spectra (Figure 2a) at this temperature, it can be inferred that photoexcitation takes place entirely through the band gap of the orthorhombic phase (at about $1.69 \mathrm{eV}$ ), even though the dominant emission is related to the band gap of the tetragonal phase (at about $1.60 \mathrm{eV}$ ). That is because quantifiable excitation through this transition at about $1.6 \mathrm{eV}$ is not possible as the comparison of the emission and excitation spectrum suggests. ${ }^{14}$ This implies that despite the small relative volume fraction of low-energy states, these sites offer the predominant radiative exciton recombination pathway. ${ }^{14}$ From the comparison of the emission and excitation spectra, it can be deduced that small individual crystallites could cause a high density of subgap states at the phase boundaries. In fact, we can infer from Figure 6 that the grain size determines which band has more weight for a fixed high power. Thus, increasing the grain size from 80 to $352 \mathrm{~nm}$, it is possible to enhance the tetragonal band versus the orthorhombic band. Indeed, for grain sizes greater than 352 $\mathrm{nm}$, we would be able to eliminate the orthorhombic band. At the same time, a grain size of $100 \mathrm{~nm}$ is the optimum size to obtain the same weight in both bands (for a high power). This dependence on the grain size of the structure stability has been already observed in other materials. ${ }^{45}$ Hence, although traditionally the temperature of $165 \mathrm{~K}$ has been associated as the tetragonal-to-orthorhombic phase transition temperature, we can see in Figure 6 that the increase in the grain size allows slowing down the phase transition. On the other hand, the thickness of the perovskite layer has no influence on the appearance or disappearance of the dual emission. However, the thickness of the layer is a much more important factor than the size of the grains in the location of the energy of the band gap (see Figure 6).

3.3. Deconvoluted Gaussian Fitting. 3.3.1. Band Gap Energy. A fundamental key to improving the conversion efficiency is to better understand the yet unclear exciton recombination pathways. ${ }^{46}$ For this reason and due to the still existing contradictions when trying to interpret the PL emissions, a detailed Gaussian fitting deconvolution is necessary. This can help to give insight not only into the origin of the dual emission peak but also into the existence or not of phonon-assisted indirect transitions causing the tetragonal emission. It has been observed that the intensity of the orthorhombic band decreases with increasing temperature, whereas the intensity of the tetragonal band progressively increases. Some authors have interpreted that the PL tetragonal-band emission stems from phonon-assisted indirect transitions during the exciton recombination process, whereas the PL orthorhombic-band emission originated from direct transitions. ${ }^{47}$ Other authors, however, defend that the PL emission of the tetragonal band, which is observable for the entire temperature range, cannot be ascribed to the indirect band gap emission affected by a quantum confinement effect. ${ }^{48}$ In this context, the thorough deconvolution of our timeintegrated temperature-dependent PL bands into Gaussian peaks could provide information on the exact number of PL emissions of $\mathrm{CH}_{3} \mathrm{NH}_{3} \mathrm{PbI}_{3}$ perovskite films and their characteristics and allow to correctly interpret them.

Keeping that in mind, we have deconvoluted the measured time-integrated PL spectra profiles by using a Gaussian fitting, as shown in Figure 7 . The spectra at a few selected temperatures for each fluence are shown. The typical PL profile for the large-grain film can be divided into several Gaussian peaks, as illustrated in Figure 7. The different colors used for each of the deconvolution peaks are associated with the different symmetries defining the perovskite structures (see 
$370 \mathrm{~nJ} / \mathrm{cm}^{2} \quad 23.8 \mathrm{~nJ} / \mathrm{cm}^{2} \quad 1.43 \mathrm{~nJ} / \mathrm{cm}^{2}$

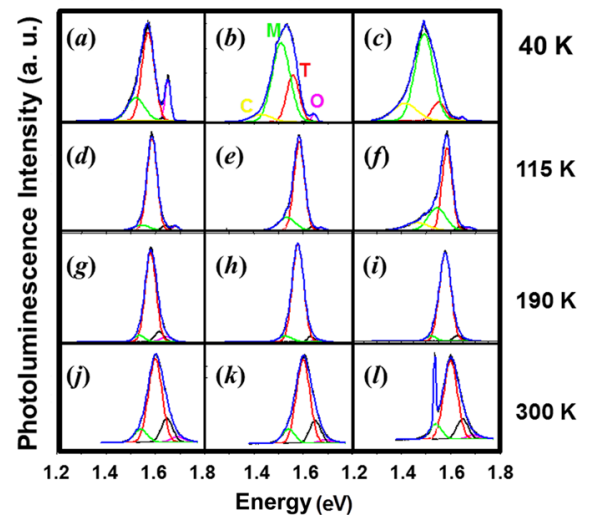

Figure 7. Deconvoluted time-integrated PL spectra profiles for the large-grain film by using Gaussian fitting. Each row shows the profiles, depending on the power, at a given temperature associated with each of the four different phases of the perovskite. Peaks assignment: pink (orthorhombic), red (tetragonal), green (low-symmetry monoclinic/ rhombohedral), and yellow (C).

Figure 7 caption). Therefore, for high fluence and temperatures below $115 \mathrm{~K}$, we can asseverate that the tetragonal and orthorhombic phases coexist (Figure 7a). This structure coexistence scenario clarifies the presence of the tetragonal peak throughout the complete temperature range studied (15$300 \mathrm{~K})$. Alternatively, this tetragonal-orthorhombic coexistence might correspond to a consequence of transitions between different points on the Fermi surface. ${ }^{26}$

To analyze and understand the meaning of the different peaks, we make use of Figure 8, where all values for the band gap energy and full width at half-maximum (fwhm) are plotted. From Figure 8a, it can be seen that there are three different temperatures for which the fwhm changes its tendency (45.5, 165 , and $300-272 \mathrm{~K})$. Some of these temperatures have been
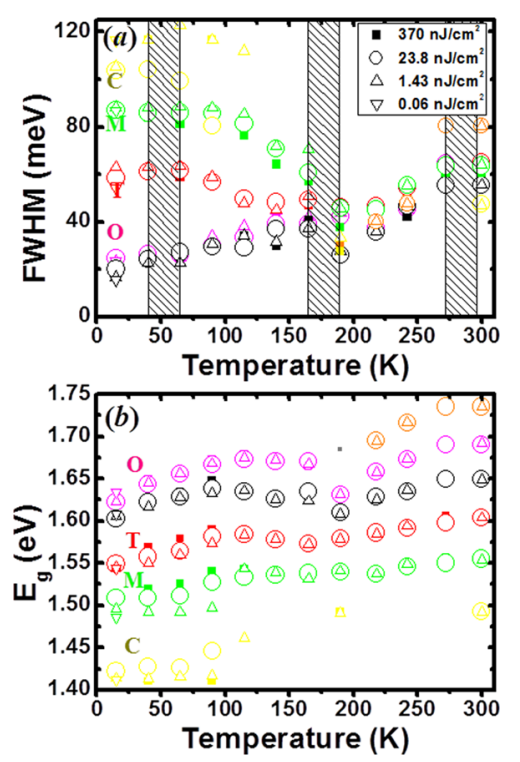

Figure 8. Temperature-dependent data of the (a) fwhm and (b) band gap energy of the different peaks obtained from the PL spectra by using deconvoluted Gaussian fitting. Note that the orthorhombic structure has a larger band gap than the tetragonal structure, as theoretical calculations have already revealed. ${ }^{27}$ The uncertainty of the data is less than the size of the symbol. already associated with phase transitions previously reported in the literature. For example, Weller et al. ${ }^{49}$ explored the complete structure of $\mathrm{MAPbI}_{3}$ by using neutron powder diffraction for the temperature range between 100 and $352 \mathrm{~K}$, which has yielded key evidences on light atom locations in this heavy metal compound. ${ }^{49}$ Thus, $300-272 \mathrm{~K}$ corresponds to the phase transition from cubic to tetragonal, ${ }^{49}$ whereas $165 \mathrm{~K}$ corresponds to the transition of phases from tetragonal to orthorhombic. ${ }^{49}$ Hence, it can be deduced that $45.5 \mathrm{~K}$ could also be associated with a new structural transformation/phase transition. That is to say, the temperature dependence of the peak positions and widths suggests the existence of a novel phase below $45.4 \mathrm{~K}$, which has not been previously reported in the literature. Hence, the existence of this possible new polymorph remains to be proven. Others arguments in favor of our observation are as follows: (i) The structural systematics in the isostructural compound under room conditions for $\mathrm{MAGeCl}_{3}$ evidence the existence of four different phases, cubic, trigonal, orthorhombic, and monoclinic, going down in temperature. (ii) It is known that the $\left\{\mathrm{PbI}_{3}\right\}^{-}$framework becomes less distorted with the increase of temperature, whereas the $\mathrm{MA}^{+}$cation experiences larger atomic translations and displaces leisurely to the center of the perovskite cube, causing the creation of the cubic phase at $327 \mathrm{~K}^{49}$ The freely rotating $\mathrm{MA}^{+}$cation on the picosecond timescale (typical of the cubic structure) is progressively detained by decreasing the temperature. Hence, for the tetragonal structure, there exist only eight disordered $\mathrm{MA}^{+}$states, ${ }^{49,50}$ whereas in the orthorhombic (Pnma) structure, the cations are fixed. Quarti et al. ${ }^{51}$ explored the stable $\mathrm{MA}^{+}$cation orientation reachable within $0.1 \mathrm{eV}$ per unit cell by using polar and apolar structural models. This study showed a variety of stable structures. This fact supports the existence of domains with different orientations prevailing within the same crystal. ${ }^{49,51}$

Keeping this in mind, we suggest that, by decreasing the temperature, an extra symmetry lowering takes place leading to the new phase. This new structure, which forms around $45.5 \mathrm{~K}$, should be identifiable from X-ray diffraction but probably difficult to determine because of the existence of multiple twin domains. Hence, the existence of the latter low-symmetry for $T$ $<45.5 \mathrm{~K}$ cannot be discarded. Moreover, our results suggest that PL is as a suitable method to explore the structural phase transitions in organometal-halide perovskites.

At this point, we will analyze the evolution of the line width (fwhm; $\Gamma$ ) with the temperature (see Figure $8 \mathrm{a}$ ), keeping in mind that the independent Boson model is no longer valid: ${ }^{52}$ (i) At low temperatures $(T<45.5 \mathrm{~K})$, we would obtain the inhomogeneous broadening contribution $\left(\Gamma_{0}=74.4 \mathrm{meV}\right)$. This term dominates at relatively low temperatures, and a nearly constant broadening was expected. This result is larger than that obtained in a less precise way, where a value of 38 $\mathrm{meV}$ was obtained, ${ }^{26}$ whereas it is much more similar to the behavior described more recently by Dar et al. ${ }^{6}$ (ii) While raising the temperature from 45.5 to $165-218 \mathrm{~K}$, it was found that the line width decreases (increases) almost linearly with temperature for the C-, M-, and T-peaks ( $\mathrm{O}-\mathrm{N}$-peaks). At this point, it is expected that acoustic $(\sigma)$ and optical phonon $\left(\Gamma_{\text {op }}\right)$ contributions dominate. The maximum value of both contributions to width broadening corresponds to $70 \mathrm{meV}$ and varies between $\sigma+\Gamma_{\text {op }}=70$ and $40.4 \mathrm{meV}$, depending on the temperature. (iii) At temperatures above 165-218 K, an increase of the line width with temperature is measured. The contribution to width broadening from the acoustic phonons 
can be neglected, ${ }^{26}$ and the optical phonon energy takes a value of $\Gamma_{\text {op }}=27.0 \mathrm{meV}$. This outcome is consistent with the Raman scattering experiment on $\mathrm{CH}_{3} \mathrm{NH}_{3} \mathrm{PbI}_{3}$, which revealed optical phonons with an energy of $25-42 \mathrm{meV}$ for the torsion mode of the organic cations, whereas the vibration of the inorganic cage and the vibration of the organic cations are associated with much smaller energies. ${ }^{26}$

Moreover, it can be demonstrated that the shift of the line width $(\Delta$ fwhm $)$ scales with the fwhm, although this dependence varies in each one of these three already exposed regions. It is necessary to note that $\Gamma_{0}=74.4 \mathrm{meV}$ is the inflection value that causes the line width to decrease or increase with temperature. For a linewidth $<74.4 \mathrm{meV}(>74.4 \mathrm{meV})$, the line width will increase (decrease) with increasing temperature. This variation will be larger as we move away from fwhm $\mathrm{m}_{0}$.

As shown in Figure 5, when studying the band gap energy, we have distinguished three blocks (high, low, and sublow). Further in-depth analyses of the PL spectra reveal that they can be better described by five to six peaks, as obtained from Gaussian fit deconvolution (Figure $8 \mathrm{~b}$ and Table 2). These peaks will be here labelled orthorhombic, tetragonal, lowsymmetry monoclinic/rhombohedral, and " $\mathrm{C}$ ", from higher to lower energies and are shown in Figure $8 \mathrm{~b}$. All these peaks behave similarly during heating. Both the lower energy emission peak attributable to the tetragonal phase $(\mathrm{T})$ and the highest energy emission peak attributable to the orthorhombic phase $(\mathrm{O})$ of $\mathrm{CH}_{3} \mathrm{NH}_{3} \mathrm{PbI}_{3}$ experience twice the blue shift previously reported. ${ }^{6}$ Additionally, a continuous blue shift for temperatures higher than $165 \mathrm{~K}$ has also been observed. Both the blue shifts can be described by a temperature coefficient $\alpha=\partial E / \partial T$ (see Table 3 ). Wu et

Table 3. Temperature Coefficient of $\mathrm{CH}_{3} \mathrm{NH}_{3} \mathrm{PbI}_{3}$ Obtained for Different Temperature Regions

\begin{tabular}{ccccc} 
& \multicolumn{4}{c}{$\alpha=\partial E / \partial T(\mu \mathrm{eV} / \mathrm{K})$} \\
\cline { 2 - 5 }$T(\mathrm{~K})$ & orthorhombic & tetragonal & low-symmetry & $\mathrm{C}$ \\
45.5 & $396(30)$ & $455(14)$ & $291(30)$ & $147(20)$ \\
165 & $-164(50)$ & $-168(30)$ & $13(60)$ & \\
300 & $535(50)$ & $235(13)$ & $147(17)$ & $236(19)$ \\
\hline
\end{tabular}

al. $^{26}$ gave only a value of $0.30 \mathrm{meV} / \mathrm{K}$ for $\alpha$ for the tetragonal structure (and for high temperature), which is similar to that obtained by us $(0.268 \mathrm{meV} / \mathrm{K})$. Additionally, we have obtained $\alpha$ for all different peaks, and they define three clear temperature regimes: (i) blue shift between 45.5 and $115 \mathrm{~K}$, (ii) red shift between 115 and $165 \mathrm{~K}$, and (iii) another blue shift between 165 and $272 \mathrm{~K}$. Hence, while increasing the temperature from 115 to $165 \mathrm{~K}$, the whole of the peaks reveal a systematic red shift, whereas an unusual blue shift is observed for a temperature higher than $165 \mathrm{~K}$. The blue shift between 165 and $272 \mathrm{~K}$ is probably connected with the opposite effects on the band gap energy of the relationship between the thermal expansion and the electron-phonon renormalization, ${ }^{26}$ as in IV-VI semiconductors (e.g., PbS, PbSe, and $\mathrm{PbTe}) .^{53}$ Its disagreement with the empirical Varshni ${ }^{54}$ Pässler ${ }^{55}$ and Bose-Einstein ${ }^{56}$ models of the standard tetrahedral semiconductor has already been reported. However, so far, this blue shift behavior has also been measured in nonconventional semiconductors, such as lead/copper chalcogenide semiconductors. ${ }^{57}$ Furthermore, it can be demonstrated that the blue/red shift $\left(\Delta E_{\mathrm{g}}\right)$ scales linearly with $E_{\mathrm{g}}$. Thus, we obtain the value of the fundamental perovskite band gap to be
$E_{\mathrm{g} 0}(45.5 \mathrm{~K})=1.38 \mathrm{eV}$, which is the ideal value for singlejunction photovoltaic applications. Thus, $\mathrm{CH}_{3} \mathrm{NH}_{3} \mathrm{PbI}_{3}$ is found to possess a direct band gap of $1.38 \mathrm{eV}$. Moreover, fundamental perovskite band gap energies $\left(E_{\mathrm{g} 0}\right)$ at 165 and $300 \mathrm{~K}$ are 1.47 and $1.44 \mathrm{eV}$, respectively.

3.3.2. Binding Energy. Much has been conjectured about the great disparity of the estimated values of binding energy $E_{\mathrm{B}}$. To clarify this point, in Figure 9 we have represented the peak

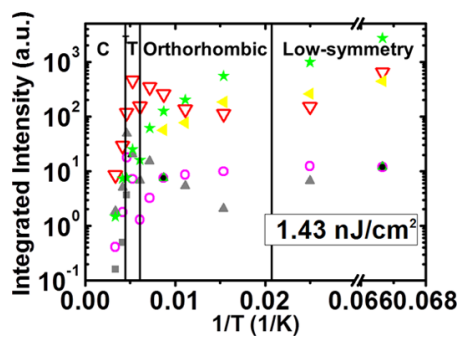

Figure 9. Temperature-dependent data of integrated intensity at the laser excitation power of $1.43 \mathrm{~nJ} / \mathrm{cm}^{2}$. Phase transitions are associated with the local minima. The uncertainty of the data is less than the size of the symbol.

integrated intensities as a function of $1 / T$. Two distinct behaviors are observed. The tetragonal-orthorhombic transition temperature separates both regimes. Moreover, minimum values of the integrated intensity are obtained at $T=45$, 165 , and $280 \mathrm{~K}$. To fit this temperature-dependent intensity associated to free exciton dissociation, we have used the following equation ${ }^{58,59}$

$$
I(T)=\frac{I_{0}}{1+A \mathrm{e}^{-E_{\mathrm{B}} / k_{\mathrm{B}} T}}
$$

in which $I_{0}$ is the intensity at $0 \mathrm{~K}, \mathrm{~A}$ the rate constant of the exciton dissociation process and $k_{\mathrm{B}}$ is the Boltzmann constant.

First of all, we made the fitting obviating the difference of behavior before and after the transition temperature. In that way, we have estimated the fluence dependence of $E_{\mathrm{B}}$ for three of these peaks: tetragonal-, orthorhombic-, and low-symmetrystructures. Thus, an increase in $E_{\mathrm{B}}$ is observed with the decrease in power. The values obtained for the orthorhombic phase are concentrated between $42 \mathrm{meV}\left(370 \mathrm{~nJ} / \mathrm{cm}^{2}\right)$ and 56 $\mathrm{meV}\left(1.43 \mathrm{~nJ} / \mathrm{cm}^{2}\right)$, whereas for the tetragonal phase, they vary between $25 \mathrm{meV}\left(370 \mathrm{~nJ} / \mathrm{cm}^{2}\right)$ and $61 \mathrm{meV}(1.43 \mathrm{~nJ} /$ $\mathrm{cm}^{2}$ ). The highest values obtained for the tetragonal phase are close to those published by some authors. For example, for the $\mathrm{CH}_{3} \mathrm{NH}_{3} \mathrm{PbI}_{3}$ film, the published $E_{\mathrm{B}}$ values are $62.3 \mathrm{meV}$ from the PL measurements ${ }^{26}$ and $55 \mathrm{meV}$ from the absorption data $^{26}$ or $50 \mathrm{meV}$ from the magneto-absorption data. ${ }^{60}$ Nevertheless, we should note that this exciton binding energy was obtained without taking into account the two different behaviors before and after the transition temperatures; therefore, this exciton binding energy may be overestimated, being the upper bound for $\mathrm{E}_{\mathrm{B}}$ of halide perovskites.

It would be more accurate to differentiate for temperatures lower and higher than the transition temperature. Thus, lower exciton binding energy values are obtained for the tetragonal structure (see Table 4). In the region where the orthorhombic phase dominates, the activation energy at high fluence excitation $(24 \mathrm{meV})$ is similar to the exciton binding energy obtained from the optical absorption measurements, 29(6) $\mathrm{meV}$ (see section 3.1). Similarly, in the region where the tetragonal phase dominates, the binding energy $(6-11 \mathrm{meV})$ 
Table 4. Binding Energy

\begin{tabular}{|c|c|c|c|c|c|c|}
\hline \multirow[b]{3}{*}{ power $\left(\mathrm{nJ} / \mathrm{cm}^{2}\right)$} & \multicolumn{6}{|c|}{$E_{\mathrm{B}}(\mathrm{meV})$} \\
\hline & \multicolumn{2}{|c|}{ sub-low } & \multicolumn{2}{|c|}{ orthorhombic } & \multicolumn{2}{|c|}{ tetragonal } \\
\hline & $T<165 \mathrm{~K}$ & $T>165 \mathrm{~K}$ & $T<165 \mathrm{~K}$ & $T>165 \mathrm{~K}$ & $T<115 \mathrm{~K}$ & $T>115 \mathrm{~K}$ \\
\hline 370 & $39(12)$ & $5(6)$ & $24(16)$ & $3(4)$ & $7(5)$ & $11(7)$ \\
\hline 23.8 & 147(30) & $9(5)$ & $57(14)$ & $3(2)$ & $13(7)$ & $9(4)$ \\
\hline 1.43 & $482(80)$ & $12(5)$ & $70(20)$ & $2(2)$ & $30(11)$ & $6(4)$ \\
\hline
\end{tabular}

also agrees with the value deduced from optical absorption in section $3.1(13(5) \mathrm{meV})$.These results also agree with values of $18-24 \mathrm{meV}^{27}$ or $32 \mathrm{meV},{ }^{14,59}$ already reported for the tetragonal structure by using microwave photoconductance and PL experiments. The present study suggests that for the same composition, the exciton properties of hybrid perovskites are sensitive to the fluence, temperature, synthesis, and structure details, such as the grain size. As probed, at high temperature, the exciton screening (due to collective orientational motion of the $\mathrm{MA}^{+}$cations) produces a large population of free carriers. ${ }^{27}$ Another possibility for the interpretation of the activation energies listed in Table 4 for sub-low and orthorhombic phases is to consider that they are associated to a carrier detrapping mechanism, instead of exciton dissociation.

3.3.3. Excitonic Character of the Peaks. The fluence-PL dependence was additionally conducted in the range of $0.06-$ $370 \mathrm{~nJ} / \mathrm{cm}^{2}$. The position and intensity of the different PL bands/peaks also vary with laser fluences because the inherent band gap of $\mathrm{MAPbI}_{3}$ increases with the accumulation of charges during photoexcitation. ${ }^{6,61}$ With increasing fluence at temperatures below $90 \mathrm{~K}$, a continuous blue shift of the lowenergy emission band (tetragonal- and low-symmetry-phase) is observed because of the tetragonal band-filling effect. ${ }^{61}$ Deconvolution shows how the tetragonal peak fills with fluence excitation, whereas the low-symmetry peak " $M$ " decreases (see Figure $7 a-c$ ), causing the blue shift of the low-energy emission band. Indeed, once the tetragonal peak has been filled, the orthorhombic peak begins to increase. At high fluence, the coexistence of the tetragonal phase and the orthorhombic phase is observed. Figure $8 \mathrm{~b}$ shows the different peaks that undergo a blue shift with the fluence increase, being greater for the peaks assigned to smaller $E_{\mathrm{g}}$. By contrast, only at higher fluences $\left(>200 \mathrm{~nJ} / \mathrm{cm}^{2}\right)$, the orthorhombic peak shows a blue shift. This could be explained if the dynamic charge transfer from the orthorhombic to tetragonal structure takes place at lower fluences $\left(<200 \mathrm{~nJ} / \mathrm{cm}^{2}\right)$. Hence, the band-filling effect dominates at higher fluences $\left(>200 \mathrm{~nJ} / \mathrm{cm}^{2}\right)$.

At temperatures lower than $45.5 \mathrm{~K}$, a perceptible sublinear dependence of the integrated PL intensity on the fluence was detected in the $E_{\mathrm{g}}$ position of the "C" peak obtained by deconvolution, whereas the low-symmetry peak " $M$ " was almost linear (see Figure 10). This sublinear dependence observed for $T<40 \mathrm{~K}$ can be produced by the inefficient diffusion of the photogenerated carriers and suggests the presence of nonradiative recombination at low temperatures (see Figure 11). By contrast, the rest of the peaks display an overlinear relationship between the fluence and the integrated PL intensity. At higher temperatures, the "C" peak disappears, whereas the rest of the peaks go closer to a biexcitonic behavior, as shown in Figure 10. An increment in the carrier density yields the saturation of nonradiative recombination centers, getting better effective internal quantum efficiency and suggesting that Auger recombination plays a minimal role under this fluence. ${ }^{6}$

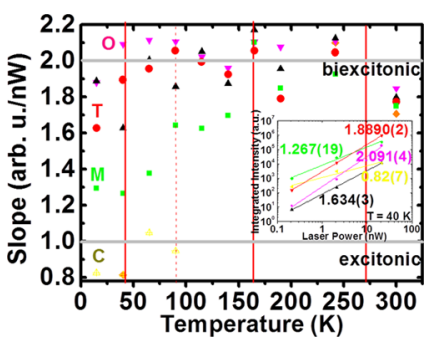

Figure 10. Excitonic character of the peaks: "C" (exciton, $\mathrm{X}$ ), tetragonal (biexciton, 2X), low-symmetry " $\mathrm{M}$ " (Trion+, $\mathrm{X}^{+}$), and orthorhombic $\left(2 \mathrm{X}^{+}\right)$peaks. Inset: Excitation fluency-dependent data of integrated intensity at the temperature of $40 \mathrm{~K}$; this was used to determine each of the values of the slopes plotted in Figure 10.

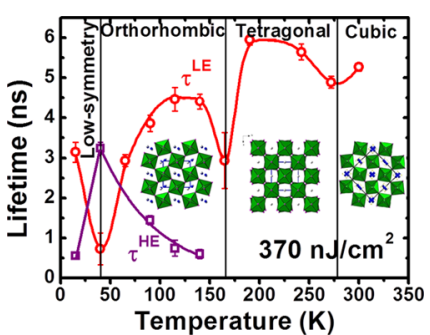

Figure 11. Radiative lower energy $\left(\tau^{\mathrm{LE}}\right)$ and higher energy $\left(\tau^{\mathrm{HE}}\right)$ lifetime versus temperature for the excitation fluencies of $370 \mathrm{~nJ} / \mathrm{cm}^{2}$. Inset: Structural stability of $\mathrm{CH}_{3} \mathrm{NH}_{3} \mathrm{PbI}_{3}$ as a function of temperature.

From Figure 10, it can also be deduced that one C-exciton ("C" peak) exists in our larger grain perovskite film. On the one side, the spin-orbit splitting of the valence band could produce another different exciton, M-exciton (low-symmetry peak " $M$ "). Because of Figure $8 \mathrm{~b}$, in which the optical gap for $\mathrm{C}$-exciton is smaller, the hole of the C-exciton (M-exciton) should be situated in the upper (lower) branch of the valence band. However, this M-exciton (low-symmetry peak "M") also could be understood as a positive Trion, in which the exciton is bound to a hole. In our PL spectrum of Figure $7 b, c$, it can be observed that the low-symmetry "M" (Trion, $\mathrm{X}^{+}$) peak is stronger than the "C" (exciton, $\mathrm{X}$ ) and tetragonal (biexciton, 2X) peaks (see Figure 10). This could be a signature of an extrinsic doped monolayer, arising from the charged trap states present in the $\mathrm{SiO}_{2}$ substrate. The orthorhombic phase, which has been detected at higher temperature and excitation fluence, is associated with $2 \mathrm{X}^{+}$.

3.4. Time-Resolved PL. TRPL emission was measured by means of time-correlated single-photon counting at different temperatures, excitation fluencies, and emission energies. From these measurements, the life time is calculated that can be seen in Figure 11. At an excitation fluence of $370 \mathrm{~nJ} / \mathrm{cm}^{2}$ (see Figure 11), the radiative emission of the lower energy band (red line/circle) and the higher energy band (orthorhombic peak, purple line/square) has been calculated. The radiative 
emission shows clearly three drops of the lifetime with the temperature, which confirm the temperatures of the three phase transitions already determined by time-integrated PL. In addition, there is a concordance with the temperatures at which the minimum values are observed in the integrated intensity versus $1 / T$ of Figure 9 . From Figure 11, it can also be seen that in all phase transitions (at $165 \mathrm{~K}$ and around $280 \mathrm{~K}$ ), only the radiative emission takes place, with the exception of the transition that takes place at the temperature close to 45.5 $\mathrm{K}$ (in which both the radiative and nonradiative emissions could be involved, as suggested from Figure 10). As aforementioned, our results suggest that a phase transition from a low-symmetry to an orthorhombic phase occurs at that temperature where the symmetry of the anionic sublattice is modified, whereas no ostensible modification of the cation dynamics is suggested. By using a three-level model with thermal activation energies $\left(E_{\text {act }}\right)$ of 53.63(12), 45.67(15), and $50.86(14) \mathrm{meV}$, depending on the PL components, we can explain a possible nonradiative emission at $45.5 \mathrm{~K}$ by $\mathrm{PL}$ quenching due to the thermal escape of electrons. This would explain why when increasing the temperature to $140 \mathrm{~K}$ there is a decrease in the intensity of the peaks associated with $2 \mathrm{X}^{+}$ (orthorhombic) and $\mathrm{X}^{+}$(monoclinic/rhombohedral) versus the increase observed for $2 \mathrm{X}$ (tetragonal) (see Figure 7). Additionally, dark states of photoexcited carriers are revealed with the activation energies due to thermal excitation $\left(E_{\text {low }}\right)$ of 41(2), 30.5(7), and 35.06(14) meV. By contrast, these activation energies are too low if we compare it to the onset of the reorientation of the pyramidal $\mathrm{GeCl}_{3}^{-}$anion (627 $\mathrm{meV}){ }^{62}$ and no electron tunneling is observed. Thus, observing the inset of Figure 11, it is clear that halogen (I) shifts with the decrease in the temperature while metals do not $(\mathrm{Pb})$. This may remind the previously exposed ideas of displacements of anions $\left(\mathrm{O}_{2}\right)$ against the stillness of metals by applying high pressures to certain oxides. In this way, it could be difficult to recognize a phase transition by X-rays when looking at the positions of the metals $(\mathrm{Pb})$ if they do not suffer appreciable displacements. Other techniques, such as Raman that are sensitive to the vibrations of oxygen (halogens) or transient PL measurements, can give us information of possible phase transformations that take place. In that way, we could discriminate between defects and phase transitions. Therefore, it is necessary to note that contrary to what has been argued, ${ }^{6}$ the importance of the halide ion ( $\mathrm{I}, \mathrm{Br}$ ) should not be underestimated.

\section{CONCLUSIONS}

We have reported the optical absorption and PL emission characteristics of $\mathrm{CH}_{3} \mathrm{NH}_{3} \mathrm{PbI}_{3}$ perovskite as a function of several important parameters such as the grain size, temperature, excitation fluencies wavelength, and time (in the case of TRPL measurements). This systematic and detailed study allows us to clarify the nature of the not well-known dual emission peak, finding absorption and emission tendencies and suggesting the existence of a novel low-temperature phase with symmetry reduction. Sometimes, for convenience, it can be assumed that the vibration of each group of bands is independent of the rest of the molecule or compound, but we should bear in mind that those vibrations can be strongly coupled. This fact makes techniques such as Raman and IR spectroscopies or transient PL measurements suitable sources of information of the atomic local environment and therefore sensitive to structural phase transitions. Thus, for instance,
TRPL emission data collected in Figure 11 evidence a phase transition for this $\mathrm{CH}_{3} \mathrm{NH}_{3} \mathrm{PbI}_{3}$ compound at temperatures close to $45 \mathrm{~K}$. (More other evidences are given along the manuscript). X-ray diffraction measurements, which provide the average structure of the sampled compound, are limited for systems such as that of this perovskite because the fixing of the atomic coordinates of the light elements of the cation are responsible of the symmetry lowering at low temperatures. This reduction of symmetry below $45 \mathrm{~K}$ would also explain the increase of the number of bands.

\section{AUTHOR INFORMATION}

\section{Corresponding Author}

*E-mail: raquel.chulia@uv.es.

\section{ORCID}

Raquel Chulia-Jordan: 0000-0003-4289-0323

Alfredo Segura: 0000-0002-9979-1302

Juan Bisquert: 0000-0003-4987-4887

\section{Author Contributions}

All authors have given approval to the final version of the manuscript.

\section{Notes}

The authors declare no competing financial interest.

\section{ACKNOWLEDGMENTS}

This work has received funding from the European Union's Horizon 2020 research and innovation programme under the Marie Sklodowska-Curie grant agreement no. 704998 (R.C.J.). Financial support by the Ministerio de Economia y Competitividad (MINECO) from Spain under projects MAT2013-47192-C3-1-R, TEC2014-53727-C2-1-R and TEC2017-86102-02-1-R are also acknowledged.

\section{REFERENCES}

(1) Dawson, J. A.; Naylor, A. J.; Eames, C.; Roberts, M.; Zhang, W.; Snaith, H. J.; Bruce, P. G.; Islam, M. S. Mechanisms of Lithium Intercalation and Conversion Processes in Organic-Inorganic Halide Perovskites. ACS Energy Lett. 2017, 2, 1818-1824.

(2) Grivas, C.; Pollnau, M. Organic solid-state integrated amplifiers and lasers. Laser Photonics Rev. 2012, 6, 419-462.

(3) Kagan, C. R.; Mitzi, D. B.; Dimitrakopoulos, C. D. Organicinorganic hybrid materials as semiconducting channels in thin-film field-effect transistors. Science 1999, 286, 945-947.

(4) Stranks, S. D.; Snaith, H. J. Metal-halide perovskites for photovoltaic and light-emitting devices. Nat. Nanotechnol. 2015, 10, 391-402.

(5) Vicente, N.; Garcia-Belmonte, G. Methylammonium lead bromide perovskite battery anodes reversibly host high li-ion concentrations. J. Phys. Chem. Lett. 2017, 8, 1371-1374.

(6) Dar, M. I.; Jacopin, G.; Meloni, S.; Mattoni, A.; Arora, N.; Boziki, A.; Zakeeruddin, S. M.; Rothlisberger, U.; Grätzel, M. Origin of unusual bandgap shift and dual emission in organic-inorganic lead halide perovskites. Sci. Adv. 2016, 2, e1601156.

(7) Xing, G.; Mathews, N.; Sun, S.; Lim, S. S.; Lam, Y. M.; Gratzel, M.; Mhaisalkar, S.; Sum, T. C. Long-Range Balanced Electron- and Hole-Transport Lengths in Organic-Inorganic CH3NH3PbI3. Science 2013, 342, 344-347.

(8) Stranks, S. D.; Eperon, G. E.; Grancini, G.; Menelaou, C.; Alcocer, M. J. P.; Leijtens, T.; Herz, L. M.; Petrozza, A.; Snaith, H. J. Electron-hole diffusion lengths exceeding 1 micrometer in an organometal trihalide perovskite absorber. Science 2013, 342, 341344.

(9) Huang, W.; Yue, S.; Liu, Y.; Zhu, L.; Jin, P.; Wu, Q.; Zhang, Y.; Chen, Y.; Liu, K.; Liang, P.; Qu, S.; Wang, Z.; Chen, Y. Observation 
of Unusual Optical Band Structure of $\mathrm{CH} 3 \mathrm{NH} 3 \mathrm{PbI} 3$ Perovskite Single Crystal. ACS Photonics 2018, 5, 1583-1590.

(10) Xing, G.; Mathews, N.; Lim, S. S.; Yantara, N.; Liu, X.; Sabba, D.; Grätzel, M.; Mhaisalkar, S.; Sum, T. C. Low-temperature solutionprocessed wavelength-tunable perovskites for lasing. Nat. Mater. 2014, 13, 476-480.

(11) Fang, H.-H.; Raissa, R.; Abdu-Aguye, M.; Adjokatse, S.; Blake, G. R.; Even, J.; Loi, M. A. Photophysics of organic-inorganic hybrid lead iodide perovskite single crystals. Adv. Funct. Mater. 2015, 25, $2378-2385$.

(12) Kong, W.; Ye, Z.; Qi, Z.; Zhang, B.; Wang, M.; Rahimi-Iman, A.; $\mathrm{Wu}, \mathrm{H}$. Characterization of an abnormal photoluminescence behavior upon crystal-phase transition of perovskite $\mathrm{CH} 3 \mathrm{NH} 3 \mathrm{PbI}$. Phys. Chem. Chem. Phys. 2015, 17, 16405-16411.

(13) Panzer, F.; Baderschneider, S.; Gujar, T. P.; Unger, T.; Bagnich, S.; Jakoby, M.; Bässler, H.; Hüttner, S.; Köhler, J.; Moos, R.; Thelakkat, M.; Hildner, R.; Köhler, A. Reversible Laser-Induced Amplified Spontaneous Emission from Coexisting Tetragonal and Orthorhombic Phases in Hybrid Lead Halide Perovskites. Adv. Opt. Mater. 2016, 4, 917-928.

(14) Wehrenfennig, C.; Liu, M.; Snaith, H. J.; Johnston, M. B.; Herz, L. M. Charge carrier recombination channels in the low-temperature phase of organic-inorganic lead halide perovskite thin films. APL Mater. 2014, 2, 081513.

(15) Yang, W. S.; Noh, J. H.; Jeon, N. J.; Kim, Y. C.; Ryu, S.; Seo, J.; Seok, S. I. High-performance photovoltaic perovskite layers fabricated through intramolecular exchange. Science 2015, 348, 1234-1237.

(16) Wolf, S. D.; Holovsky, J.; Moon, S.-J.; Löper, P.; Niesen, B.; Ledinsky, M.; Haug, F.-J.; Yum, J.-H.; Ballif, C. Organometallic halide perovskites: Sharp optical absorption edge and its relation to photovoltaic performance. J. Phys. Chem. Lett. 2014, 5, 1035-1039.

(17) Lee, M. M.; Teuscher, J.; Miyasaka, T.; Murakami, T. N.; Snaith, H. J. Efficient hybrid solar cells based on meso-superstructured organometal halide perovskites. Science 2012, 338, 643-647.

(18) Chuliá-Jordán, R.; Santamaría-Pérez, D. Four-mode plasmonic structure based on a prism-grating anticrossing bandgap. Appl. Phys. Lett. 2012, 100, 063301.

(19) Suárez, I.; Juárez-Pérez, E. J.; Bisquert, J.; Mora-Seró, I.; Martínez-Pastor, J. P. Polymer/perovskite amplifying waveguides for active hybrid silicon photonics. Adv. Mater. 2015, 27, 6157-6162.

(20) Muñoz-Matutano, G.; Barrera, D.; Fernández-Pousa, C. R.; Chulia-Jordan, R.; Martínez-Pastor, J.; Gasulla, I.; Seravalli, L.; Trevisi, G.; Frigeri, P.; Sales, S. Parallel recording of single quantum dot optical emission using multicore fibers. IEEE Photonics Technol. Lett. 2016, 28, 1257-1260.

(21) Muñoz-Matutano, G.; Barrera, D.; Fernández-Pousa, C. R.; Chulia-Jordan, R.; Seravalli, L.; Trevisi, G.; Frigeri, P.; Sales, S.; Martínez-Pastor, J. All-optical fiber hanbury brown \& twiss interferometer to study $1300 \mathrm{~nm}$ single photon emission of a metamorphic InAs quantum dot. Sci. Rep. 2016, 6, 27214.

(22) Chuliá-Jordán, R.; Unger, A. Comparison of the different bandgap cavities in a metallic four-mode plasmonic structure. Plasmonics 2015, 10, 429-438.

(23) Chuliá-Jordán, R.; Santamaría-Pérez, D. Tuning the propagation constant by the anticrossing bandgap prism coupling technique. Plasmonics 2012, 7, 665-675.

(24) Kao, T. S.; Chou, Y.-H.; Chou, C.-H.; Chen, F.-C.; Lu, T.-C. Lasing behaviors upon phase transition in solution-processed perovskite thin films. Appl. Phys. Lett. 2014, 105, 231108.

(25) Dhanker, R.; Brigeman, A. N.; Larsen, A. V.; Stewart, R. J.; Asbury, J. B.; Giebink, N. C. Random lasing in organo-lead halide perovskite microcrystal networks. Appl. Phys. Lett. 2014, 105, 151112. (26) Wu, K.; Bera, A.; Ma, C.; Du, Y.; Yang, Y.; Li, L.; Wu, T. Temperature-dependent excitonic photoluminescence of hybrid organometal halide perovskite films. Phys. Chem. Chem. Phys. 2014, 16, 22476-22481.

(27) Even, J.; Pedesseau, L.; Katan, C. Analysis of multivalley and multibandgap absorption and enhancement of free carriers related to exciton screening in hybrid perovskites. J. Phys. Chem. C 2014, 118, 11566-11572.

(28) Hirasawa, M.; Ishihara, T.; Goto, T.; Uchida, K.; Miura, N. Magnetoabsorption of the lowest exciton in perovskite-type compound (CH3NH3)PbI3. Phys. Rev. B: Condens. Matter Mater. Phys. 1994, 201, 427-430.

(29) Hsiao, Y.-C.; Wu, T.; Li, M.; Liu, Q.; Qin, W.; Hu, B. Fundamental physics behind high-efficiency organo-metal halide perovskite solar cells. J. Mater. Chem. A 2015, 3, 15372-15385.

(30) von der Osten, W.; Stolz, H. Localized exciton states in silver halides. J. Phys. Chem. Solids 1990, 51, 765-791.

(31) Williams, R. T.; Song, K. S. The self-trapped exciton. J. Phys. Chem. Solids 1990, 51, 679-716.

(32) Yamada, Y.; Nakamura, T.; Endo, M.; Wakamiya, A.; Kanemitsu, Y. Near-band-edge optical responses of solutionprocessed organic-inorganic hybrid perovskite $\mathrm{CH} 3 \mathrm{NH} 3 \mathrm{PbI} 3$ on mesoporous TiO2electrodes. Appl. Phys. Express 2014, 7, 032302.

(33) Arora, N.; Dar, M. I.; Hezam, M.; Tress, W.; Jacopin, G.; Moehl, T.; Gao, P.; Aldwayyan, A. S.; Deveaud, B.; Grätzel, M.; Nazeeruddin, M. K. Photovoltaic and amplified spontaneous emission studies of high-quality formamidinium lead bromide perovskite films. Adv. Funct. Mater. 2016, 26, 2846-2854.

(34) Oku, T. Crystal Structures of $\mathrm{CH}_{3} \mathrm{NH}_{3} \mathrm{PbI}_{3}$ and Related Perovskite Compounds Used for Solar Cells. Solar Cells-New Approaches and Reviews; InTech, 2015; pp 77-101.

(35) Santamaría-Pérez, D.; Chuliá-Jordán, R. Compression of mineral barite, BaSO4: a structural study. High Pressure Res. 2011, 32, 81-88.

(36) Santamaría-Pérez, D.; Kumar, R. S.; Dos Santos-García, A. J.; Errandonea, D.; Chulia-Jordan, R.; Saez-Puche, R.; RodríguezHernández, P.; Muñoz, A. High-pressure transition to the post-barite phase in $\mathrm{BaCrO} 4$ hashemite. Phys. Rev. B: Condens. Matter Mater. Phys. 2012, 86, 094116.

(37) Santamaría-Pérez, D.; Gracia, L.; Garbarino, G.; Beltrán, A.; Chuliá-Jordán, R.; Gomis, O.; Errandonea, D.; Ferrer-Roca, C.; Martínez-García, D.; Segura, A. High-pressure study of the behavior of mineral barite by X-ray diffraction. Phys. Rev. B: Condens. Matter Mater. Phys. 2011, 84, 054102.

(38) Saito, K.; Kurosawa, T.; Akai, T.; Yokoyama, S.; Morioka, H.; Oikawa, T.; Funakubo, H. Characterization of epitaxial $\mathrm{Pb}(\mathrm{Zrx}, \mathrm{Til}-$ x)O3 thin films with composition near the morphotropic phase boundary. MRS Proc 2002, 748, U13.4.

(39) Yokoyama, S.; Honda, Y.; Morioka, H.; Oikawa, T.; Funakubo, H.; Iijima, T.; Matsuda, H.; Saito, K. Large piezoelectric response in (111)-oriented epitaxial $\mathrm{Pb}(\mathrm{Zr}, \mathrm{Ti}) \mathrm{O} 3$ films consisting of mixed phases with rhombohedral and tetragonal symmetry. Appl. Phys. Lett. 2003, 83, 2408-2410.

(40) Kelman, M. B.; McIntyre, P. C.; Hendrix, B. C.; Bilodeau, S. M.; Roeder, J. F.; Brennan, S. Structural analysis of coexisting tetragonal and rhombohedral phases in polycrystalline $\mathrm{Pb}$ (Zr0.35Ti0.65)O3 thin films. J. Mater. Res. 2003, 18, 173-179.

(41) Zeches, R. J.; Rossell, M. D.; Zhang, J. X.; Hatt, A. J.; He, Q.; Yang, C.-H.; Kumar, A.; Wang, C. H.; Melville, A.; Adamo, C.; Sheng, G.; Chu, Y.-H.; Ihlefeld, J. F.; Erni, R.; Ederer, C.; Gopalan, V.; Chen, L. Q.; Schlom, D. G.; Spaldin, N. A.; Martin, L. W.; Ramesh, R. A strain-driven morphotropic phase boundary in $\mathrm{BiFeO}$. Science 2009, 326, 977-980.

(42) Chen, Z.; You, L.; Huang, C.; Qi, Y.; Wang, J.; Sritharan, T.; Chen, L. Nanoscale domains in strained epitaxial BiFeO3 thin Films on LaSrAlO4 substrate. Appl. Phys. Lett. 2010, 96, 252903.

(43) Ehm, L.; Borkowski, L. A.; Parise, J. B.; Ghose, S.; Chen, Z. Evidence of tetragonal nanodomains in the high-pressure polymorph of BaTiO3. Appl. Phys. Lett. 2011, 98, 021901.

(44) Wehrenfennig, C.; Liu, M.; Snaith, H. J.; Johnston, M. B.; Herz, L. M. Homogeneous emission line broadening in the organo lead halide perovskite $\mathrm{CH}_{3} \mathrm{NH}_{3} \mathrm{PbI}_{3}$-xClx. J. Phys. Chem. Lett. 2014, 5, $1421-1426$. 
(45) Zhang, H.; Banfield, J. F. Thermodynamic analysis of phase stability of nanocrystalline titania. J. Mater. Chem. 1998, 8, 20732076.

(46) Chirvony, V. S.; González-Carrero, S.; Suárez, I.; Galian, R. E.; Sessolo, M.; Bolink, H. J.; Martínez-Pastor, J. P.; Pérez-Prieto, J. Delayed Luminescence in Lead Halide Perovskite Nanocrystals. J. Phys. Chem. C 2017, 121, 13381-13390.

(47) Zhang, Y.; Yin, J.; Parida, M. R.; Ahmed, G. H.; Pan, J.; Bakr, O. M.; Brédas, J.-L.; Mohammed, O. F. Direct-indirect nature of the bandgap in lead-free perovskite nanocrystals. J. Phys. Chem. Lett. 2017, 8, 3173-3177.

(48) Kiba, T.; Mizushima, Y.; Igarashi, M.; Huang, C.-H.; Samukawa, S.; Murayama, A. Temperature dependence of timeresolved photoluminescence in closely packed alignment of $\mathrm{Si}$ nanodisks with SiC barriers. Nanoscale Res. Lett. 2013, 8, 223.

(49) Weller, M. T.; Weber, O. J.; Henry, P. F.; Di Pumpo, A. M.; Hansen, T. C. Complete structure and cation orientation in the perovskite photovoltaic methylammonium lead iodide between 100 and $352 \mathrm{~K}$. Chem. Commun. 2015, 51, 4180-4183.

(50) Wasylishen, R. E.; Knop, O.; Macdonald, J. B. Cation rotation in methylammonium lead halides. Solid State Commun. 1985, 56, $581-582$.

(51) Quarti, C.; Mosconi, E.; De Angelis, F. Interplay of orientational order and electronic structure in methylammonium lead iodide: Implications for solar cell operation. Chem. Mater. 2014, 26, 6557-6569.

(52) Rudin, S.; Reinecke, T. L.; Segall, B. Temperature-dependent exciton linewidths in semiconductors. Phys. Rev. B: Condens. Matter Mater. Phys. 1990, 42, 11218-11231.

(53) Pedrueza, E.; Segura, A.; Abargues, R.; Bailach, J. B.; Chervin, J. C.; Martínez-Pastor, J. P. The effect of quantum size confinement on the optical properties of $\mathrm{PbSe}$ nanocrystals as a function of temperature and hydrostatic pressure. Nanotechnology 2013, 24, 205701.

(54) Varshni, Y. P. Temperature dependence of the energy gap in semiconductors. Physica 1967, 34, 149-154.

(55) Pässler, R. Basic model relations for temperature dependencies of fundamental energy gap in semiconductors. Phys. Status Solidi B 2001, 200, 155-172.

(56) Lautenschlager, P.; Garriga, M.; Logothetidis, S.; Cardona, M. Interband critical points of $\mathrm{GaAs}$ and their temperature dependence. Phys. Rev. B: Condens. Matter Mater. Phys. 1987, 35, 9174-9189.

(57) Dey, P.; Paul, J.; Bylsma, J.; Karaiskaj, D.; Luther, J. M.; Beard, M. C.; Romero, A. H. Origin of the temperature dependence of the band gap of $\mathrm{PbS}$ and $\mathrm{PbSe}$ quantum dots. Solid State Commun. 2013, $165,49-54$.

(58) Chen, Z.; Yu, C.; Shum, K.; Wang, J. J.; Pfenninger, W.; Vockic, N.; Midgley, J.; Kenney, J. T. Photoluminescence study of polycrystalline CsSnI3 thin films: Determination of exciton binding energy. J. Lumin. 2012, 132, 345-349.

(59) Savenije, T. J.; Ponseca, C. S.; Kunneman, L.; Abdellah, M.; Zheng, K.; Tian, Y.; Zhu, Q.; Canton, S. E.; Scheblykin, I. G.; Pullerits, T.; Yartsev, A.; Sundström, V. Thermally activated exciton dissociation and recombination control the carrier dynamics in organometal halide perovskite. J. Phys. Chem. Lett. 2014, 5, 21892194.

(60) Tanaka, K.; Takahashi, T.; Ban, T.; Kondo, T.; Uchida, K.; Miura, N. Comparative study on the excitons in lead-halide-based perovskite-type crystals $\mathrm{CH} 3 \mathrm{NH} 3 \mathrm{PbBr} 3 \mathrm{CH} 3 \mathrm{NH} 3 \mathrm{PbI}$. Solid State Commun. 2003, 127, 619-623.

(61) Manser, J. S.; Kamat, P. V. Band filling with free charge carriers in organometal halide perovskites. Nat. Photonics 2014, 8, 737-743.

(62) Yamada, K.; Mikawa, K.; Okuda, T.; Knight, K. S. Static and dynamic structures of $\mathrm{CD} 3 \mathrm{ND} 3 \mathrm{GeCl} 3$ studied by TOF high resolution neutron powder diffraction and solid state NMR. J. Chem. Soc., Dalton Trans. 2002, 2112-2118. 